It has been very freely stated by surgeons throughout the world that if gastro-enterostomy is done in stomachs in which no pyloric stenosis exists the patients are frequently worse after the operation than before. This is said to be the case, especially, if no other demonstrable gastric lesion is present. The neurotic condition of the patients, instead of the inefficiency of the surgeon, has been blamed for the bad result. I have had such bad results, and I have taught according to the usually accepted ideas, but have never been quite satisfied that the statement is a correct one. In a case where gastro-enterostomy is done for duodenal ulcer where no organic pyloric stenosis exists the ulcer heals, the pyloric spasm disappears, and then results a patient with a gastro-enterostomy stoma present in a stomach which is otherwise normal, yet it is only the rare exception in such cases to have trouble. I believe that such so-called neurotic patients with prominent gastric symptoms are subjects of some lesion distal to the pylorus, whether it be in the gall-bladder, appendix, or other part of the alimentary system, and the gastro-enterostomy will undoubtedly make many of them worse than before because the "check action" of the pylorus will then be done away with, and food, probably undigested, allowed to go where it is not wanted. (If there is irritation in any part of the alimentary tract beyond the pylorus there is apparently a "message" sent to the pylorus to hold food baek. In such cases digestion is therefore upset even to the extent of causing vomiting, when relief is obtained usually at once. If a gastro-enterostomy is done in such cases without removal of the cause of the symptoms, it is more than likely that the symptoms will be aggravated because this check action is rendered inert by food passing through the stoma.) I believe that as the principles exemplified in the valuable writings of the Mayos, Moynihan, and $\mathrm{H}$. J. Paterson become more widely known and appreciated we shall hear correspondingly less of this " bogie." I think that the acceptance of the view I wish to combat has been rather pernicious in that it has led surgeons to take an unnecessary risk by excising gastric ulcers in cases where this is unduly difficult so as to avoid the performance of a gastro-enterostomy, which of itself will, I am convinced, cause healing of the ulcer. Gastro-enterostomy should be done as a routine in such cases in order to remove permanently the local con ditions which have led to the formation of the ulcer and there fore to prevent further trouble. It matters not whether gastric ulcer is due to purely local causes or whether it is the result of a more general alteration in metabolism resulting in a vciious circle which brings about the breakdown in the stomach.

Aberdeen.

\section{INTRA-MEDULLARY LESION (? GUMMA) OF THE SPINAL CORD WITH BROWN- SEQUARD PHENOMENON AND RECOVERY.}

BY ALFRED BERNSTEIN, M.B., B.S. LOND. RESIDENT OBSTETRIC ASSISTANT, LATE HOUSE PHYSICIAN, WESTMINSTER HOSPITAL.

A FEMALE, aged 18 years, was admitted to Westminster Hospital on Dec. 8th, 1909, under the care of Dr. F. de Havilland Hall, to whom I am indebted for permission to publish this case. The patient was admitted complaining of pain in the back of the head and neck with stiffness and weakness of the right arm and leg. She first noticed stiffness in the neck about three years ago. A bout two months ago (October, 1909) there was slight pain in the back of the neck radiating over the back of the scalp, especially on the right side. There was also some numbness on the posterior right scalp. This had continued till the present. In the second week in October there was some weakness in the right arm first noticed in the shoulder, which gradually got worse and by Nov. 8th the weakness had extended down to the hand. About the same time there was weakness in the right leg, which also got steadily worse. There was no numbness, but some slight tingling in the finger-tips. The patient was a packer. She had an attack of influenza a year ago (December, 1908), and 10 months ago was greatly shocked by her father's death, and "had not been the same since." The father died from phthisis. The mother and the other children had excellent history-no syphilis, cancer, or tuberculosis.
The patient was a well-developed, intelligent, bright girl Dec. 10th : Pupils dilated, equal, and react normally. External ocular movements normal. Fundi normal. Face, palate, tongue, muscles of mastication normal. No cutaneous anæsthesia to cotiton wool. Diminution to pin-prick, heat, and cold on left side of trunk bounded by the middle line and extending as high as the second left rib in front. Includes also left lower and upper limbs. There is a slight patch of anæsthesia on right lower scalp behind. Marked weakness right upper limb at all joints. No movement impossible except extension of interphalangeal joints. Weakness more marked in distal part of limb. Moderate atrophy of interossei, thenar, and hypothenar eminences of right hand. Diaphragm, intercostals, and abdominal muscles normal. Moderate weakness of right lower limb at all joints against resistance. No movements impossible. Wasting of right arm and leg, especially deltoid and hand muscles. Gait : slight dragging of right leg. Supinator jerks : R. $>$ L. Knee-jerks : R. $++;$ L. = normal. Right patellar clonus and ankle clonus. Plantar reflexes and abdominal reflexes not elicited. Sphincters normal. No spinal rigidity or tenderness. Other systems normal.

Progress of oase.-During the next week the physical signs became more marked, and by Dec. 17th there was ankle clonus on the left side but less than on the right. Likewise left knee-jerk became exaggerated, but not so much as on the right. The atrophy of the muscles increased, especially the hand muscles, which were extremely wasted, giving wellmarked "claw-hand." About the last week in December there began to be an improvement, which steadily progressed. On Dec. 31st she had gained 2 pounds 10 ounces in weight. Right arm was slightly stronger and the fingers could be slightly extended. On Jan. 7th right arm and hand much stronger. Thenar and hypothenar eminences filling out and movements improving. No reaction of degeneration. Good reaction to faradism. K.C.C. > A.C.C. Jan. 14th: Sensation in left leg normal. There is now no ankle clonus on left, while that on right is much less sustained than before. Jan. 21st : Still steady improvement. Now only the slightest wasting and weakness of interossei. The other muscies almost normal. Walks quite well. At end of the month patient quite well, and when seen two months later showed no signs of disease and said she never felt better in her life. Lumbar puncture on Dec. 28th gave 25 c.c. of fluid under increased pressure. The fluid was normal, containing only one or two lymphocytes to 1-6 inch field, and cultures were sterile. Wassermann's reaction on Jan. 6th was negative. Unfortunately this was not done until antispecific treatment had been used some weeks, but there was never any reason to suspect either congenital or acquired syphilis, and there was little hope that the use of mercury would be followed by recovery. The treatment was inunctions of mercury together with massage, and internally potassium iodide. The mercury and iodides were well borne.

Remarks. - The lesion in this case was evidently situated in the right half of the substance of the cord, especially the anterior horn in the upper dorsal region. But the nature of the lesion is more difficult to determine. Tumours of the cord, tubercle, \&c., a plaque of disseminated sclerosis, would hardly have cleared up so rapidly after advancing so far and so quickly. Neither would anterior poliomyelitis account for all the physical signs. Hysteria is excluded by the marked rapid wasting of the intrinsic muscles of the hand and the absence of other hysterical stigmata. The rapid development of all the symptoms followed by as rapid a recovery after antisyphilitic treatment seem to point to the specific origin of the lesion, which is supported by the fact that mercury was so well borne.

Spinal gummata are very rare. Gowers and Williamson have described them in the posterior horn. They usually start in the membranes or roots, much more rarely in the substance of the cord, and there is as a rule some associated meningitis manifested by numbness and tingling in the limbs and perhaps some stiffness and pain in the neck. Unfortunately in this case lumbar puncture was not performed till more than two weeks after mercurial treatment was started. It was then quite normal fluid, though in excess and under pressure. Armstrong has reported a case of crural monoplegia with anæsthesia of opposite limb and Mott a similar case.

Bibliography.-Mott: New System of Syphilis. Williamson 